\title{
Frozen-thawed embryos generated from successful fresh IVF cycles have higher potential for favorable outcome than frozen-thawed embryos obtained from unsuccessful fresh cycles: A retrospective cohort
}

Gilad Karavani ( $\sim$ Giladk84@gmail.com )

Hadassah Medical Center

Shmuel Herzberg

Hadassah Medical Center

Natali Schachter-Safrai

Hadassah Medical Center

Henry Hillel Chill

Hadassah Medical Center

Assaf Ben-Meir

Hadassah Medical Center

Alex Simon

Hadassah Medical Center

Research article

Keywords: Fresh embryo transfer, Frozen-thawed embryo transfer, Vitrification, Slow freezing

Posted Date: March 31st, 2020

DOl: https://doi.org/10.21203/rs.3.rs-19031/v1

License: (c) (i) This work is licensed under a Creative Commons Attribution 4.0 International License.

Read Full License 


\section{Abstract}

Background: Many variables, including woman`s age, embryo quality and endometrial receptivity, influence the success rate of either fresh embryo transfer (ET) or frozen-thawed ET (FT-ET) cycles. The aim of this study is to examine whether the results of a fresh in-vitro fertilization (IVF) cycle correlates with its frozen-thawed embryos transfer outcomes.

Methods: A retrospective cohort study conducted in a tertiary medical center single IVF unit between 2014 and 2017. The study population was comprised of women who underwent fresh ET with the remaining embryos frozen using vitrification and at least one consecutive FT-ET cycle. Comparison of FT-ET cycle's details and outcomes were assessed in relation to the outcome of the preceding fresh ET cycle.

Results: A total of 599 women underwent fresh ET and at least one consecutive FT-ET cycle. Significantly higher rates of successful FT-ET cycle outcomes were observed in the group of patients with a successful fresh cycle compared to the group with an unsuccessful fresh cycle (48.9\% vs. $20.8 \%, p<0.001)$. Logistic regression demonstrated an adjusted OR of 5.02 for successful FT-ET after a successful fresh cycle.

Conclusions: Frozen-thawed embryos generated from successful fresh IVF cycles have higher potential to implant when compared to frozen-thawed embryos obtained in an unsuccessful fresh cycles.

\section{Introduction}

Accurate prognostic assessment prior to embryo transfer (ET) during in-vitro fertilization (IVF) plays an important role in protocol planning, enabling better coordination of expectations between physicians and patients. The decision regarding the number of embryos transferred in each cycle is an essential one, influenced by estimation of potential implantation rate of such embryos. The higher the probability of successful transfer, the better it is to transfer fewer embryos and to freeze the rest. In recent years, the common approach is to transfer a single embryo during each cycle in order to reduce the risk of multiple pregnancies [1]. Guidelines regarding recommended number of transferred embryos per cycle have been previously described [2]. Thus, in an attempt to achieve better cycle programing, clinicians are required to assess variables which may predict a successful treatment cycle.

The most influential predictors for achieving a pregnancy in IVF treatment are: Female age [3-7], embryo quality [8], sperm quality [9], duration of subfertility [3], basal FSH $[3,7)]$ use of intracytoplasmic sperm injection (ICSI) [4] and number of retrieved oocytes [3]. It is arguable whether patients who conceived in a fresh IVF cycle and consequently used their surplus frozen embryos generated from the same ovum pickup (OPU) experience a better outcome than patients who did not conceive in their fresh cycle and seek a frozen thawed (FT) transfer attempt [10-12].

In this analysis we retrospectively examined outcomes of FT-ET, with respect to the association between success in a fresh IVF cycle and the prognosis of the frozen thawed cycle from the same oocyte retrieval. 
We hypothesized that FT-ET cycle success rates will be higher following a successful fresh cycle compared to those consecutive to an unsuccessful fresh cycle.

\section{Materials And Methods}

This retrospective cohort study was conducted at the IVF unit of a tertiary medical center between June 2014 and December 2017. Inclusion criteria were all women 18-45 years who underwent ovarian stimulation with a consequent fresh embryo transfer as well as at least one frozen-thawed embryo transfer using embryos from the same OPU with known ET outcome. All FT embryos included were frozen using vitrification. Exclusion criteria were women who did not meet the inclusion criteria for age, for whom the method of slow freezing was used during the freezing process, or for whom there were missing data regarding the fresh or one of the frozen-thawed embryo transfer cycles.

Data was collected systematically from patient electronic records after implementing the inclusion criteria mentioned above. The study was approved by the Investigation Review Board of Hadassah Hebrew University Medical Center (IRB number 0483-17-HMO).

An IVF cycle resulting in clinical pregnancy was defined as a successful cycle. The primary outcome was clinical pregnancy. A comparison was made regarding the outcome of frozen-thawed embryo transfer cycles of women who had a successful outcome (clinical pregnancy) in their fresh ET and those who had an unsuccessful outcome in their fresh cycle. Collected data included age at fresh and frozen cycles, number of oocytes retrieved, number of mature oocytes, fertilization technique (ICSI versus standard IVF), fertilization rate, timing of embryo transfer, number of transferred embryos, embryos quality, number of frozen embryos and number of consecutive frozen cycles. Preimplantation genetic testing for aneuploidy was not performed in any of the cases included in this study. Outcome assessed was clinical pregnancy rate for each group. In addition, a sub-group analysis was performed comparing the outcomes of the first frozen-thawed embryo transfer with regard to the success of the previous fresh transfer from the same OPU.

\section{IVF protocol and ET technique}

Oocyte retrieval was performed under general anesthesia using transvaginal ultrasound guidance. Embryo quality was determined by cell number, symmetry and fragmentation and was graded " $\mathrm{A}$ ", $\mathrm{B}$ " or " $\mathrm{C}$ " according to the SART grading [13]. Embryos are cultured in a one-step medium ("SAGE 1-step" (SAGE, Al-rad medical, Nes Ziona, Israel). Fresh embryo transfer was performed on day 2, day 3, or at blastocyst stage according to patient and laboratory convenience. Embryo transfer was performed using one of two catheters - the SIVF catheter (K-Jets-7019-SIVF; Cook IVF, Eight Miles Plains, Queensland, Australia) or the Edwards-Wallace catheter (Classic Embryo Replacement Catheter; Smiths Medical, Hythe, Kent, U.K.) upon operator preference. Frozen thawed embryo transfers were performed applying an artificial cycle using exogenous estradiol and progesterone supplementation as previously described [14]. Briefly, patients started 17ß-E2 6 mg/day (Estrofem; Novo Industry A/S, Copenhagen, Denmark) given orally in 3 
divided doses on the first day of their menstrual cycle. A week later, estradiol and progestrone levels were measured. Assessment of endometrial thickness was performed using transvaginal ultrasound.. Patients then proceeded with a similar dose of Estrofem for an additional 5-13 days. Once endometrial thickness measurment reached $8 \mathrm{~mm}$, micronized progestrone pills (Utrogestan; Besins healthcare, Paris, France) were added. A dose of $200 \mathrm{mg}$ three times a day vaginally for 3 days was used. .

\section{Statistical analysis}

Testing the association between two categorical variables was carried out using either the Chi-Square test or the Fisher's exact test. Comparison of quantitative variables between the two-categories of the outcome variable was performed using the Student's t-test for the two independent groups. Univariate analysis was performed to identify factors associated with successful frozen-thawed embryo transfer. A logistic regression model was used to simultaneously assess the effect of several variables on the dichotomous dependent variable.

Variables which were found to be statistically significant and associated with the dependent variable using the univariate analysis, were entered into the multivariate logistic regression model. In the model, the significance of each of the independent variables was given, and the adjusted Odds Ratio (with its $95 \%$ confidence interval) was calculated for the entire study population. A p value of $<.05$ was considered statistically significant.

\section{Results}

The vitrification technique was used during 2014-2017 for the freezing of 2481 embryos from 599 patients. In this group, 149 (24.9\%) patients achieved clinical pregnancy during their fresh ET cycle and 450 patients had an unsuccessful fresh ET. No differences were found between the two groups except for the mean number of oocytes retrieved and mature oocytes, higher in the successful fresh cycle group $(\mathrm{p}<$ 0.05 for both, Table 1). Comparison of the day of embryos freezing demonstrated similar rates of day 2 and 3 freezing and an increased rate of day 5 freezing in the successful fresh cycle group. However, the blastocyst transfer rate did not differ between the successful and unsuccessful groups (Table 2). 
Table 1

Fresh embryo transfer cycle data according to clinical pregnancy

\begin{tabular}{|c|c|c|c|}
\hline Parameter & Successful fresh cycle & Unsuccessful fresh cycle & $P$ value \\
\hline $\mathrm{N}$ of patients & 149 & 450 & \\
\hline Age at aspiration & $34.22 \pm 5.90$ & $35.25 \pm 5.86$ & 0.067 \\
\hline $\mathrm{N}$ of oocytes retrieved & $13.50 \pm 7.36$ & $11.96 \pm 6.84$ & 0.02 \\
\hline $\mathrm{N}$ of mature oocytes & $10.76 \pm 6.14$ & $9.05 \pm 5.15$ & 0.003 \\
\hline Fertilization rate & $0.70 \pm 0.19$ & $0.72 \pm 0.20$ & 0.179 \\
\hline Use of ICSI (\%) & $79.04 \pm 22.34$ & $78.89 \pm 26.17$ & 0.953 \\
\hline Grade A embryos (\%) ${ }^{a}$ & $72.02 \pm 28.01$ & $74.09 \pm 24.84$ & 0.565 \\
\hline Grade B embryos (\%) ${ }^{a}$ & $23.21 \pm 24.86$ & $22.60 \pm 26.26$ & 0.808 \\
\hline Grade C embryos (\%) ${ }^{a}$ & $4.77 \pm 1.10$ & $3.29 \pm 0.87$ & 0.126 \\
\hline $\mathrm{N}$ of frozen embryos & $4.42 \pm 3.88$ & $4.17 \pm 3.49$ & 0.500 \\
\hline $\mathrm{N}$ of transferred fresh embryos & $1.69+0.86$ & $1.55+0.85$ & 0.081 \\
\hline \multicolumn{4}{|l|}{ Data presented as Mean \pm SD } \\
\hline \multicolumn{4}{|c|}{ ICSI, Intracytoplasmic sperm injection. } \\
\hline a Embryo quality was determi & ording to the & ding & \\
\hline
\end{tabular}


Table 2

Comparison of frozen-thawed cycles data between the successful and unsuccessful fresh cycle from the same ovum pick-up

\begin{tabular}{|llll|}
\hline Parameter & $\begin{array}{l}\text { Successful fresh } \\
\text { cycle }\end{array}$ & $\begin{array}{l}\text { Unsuccessful fresh } \\
\text { cycle }\end{array}$ & $\begin{array}{l}\text { P } \\
\text { value }\end{array}$ \\
\hline N of frozen cycles & 176 & 547 & \\
\hline Age at FT-ET & $36.05 \pm 5.70$ & $35.44 \pm 5.92$ & 0.497 \\
\hline Day of freezing & & & \\
\hline Day 2 & $17 / 178(9.6 \%)$ & $86 / 561(15.3 \%)$ & 0.055 \\
\hline Day 3 & $117 / 178(65.7 \%)$ & $391 / 561(71.5 \%)$ & 0.320 \\
\hline Day 5 & $44 / 178(24.7 \%)$ & $84 / 561(15.0 \%)$ & 0.003 \\
\hline Blastocyst transfer & $37 / 178(20.8 \%)$ & $85 / 561(15.2 \%)$ & 0.079 \\
\hline Frozen-thawed cycles & $1.18 \pm 0.49$ & $1.22 \pm 0.55$ & 0.476 \\
\hline N of transferred embryos & $1.79+0.67$ & $1.69+0.72$ & 0.141 \\
\hline Implantation rate & $105 / 299(35.1 \%)$ & $123 / 889(13.4 \%)$ & 0.001 \\
\hline Clinical pregnancy in first frozen cycle & $81 / 149(54.4 \%)$ & $86 / 450(19.1 \%)$ & $<$ \\
\hline Overall clinical pregnancies in frozen cycles & $86 / 176(48.9 \%)$ & $114 / 547(20.8 \%)$ & 0.001 \\
\hline Patients with at least 1 clinical pregnancy in a & $82 / 149(55.0 \%)$ & $103 / 450(22.9 \%)$ & 0.001 \\
\hline frozen cycle & & & \\
\hline Data presented as Mean \pm SD or n/N (\%). & & & 0.001 \\
\hline
\end{tabular}

Overall, pregnancy rate for FT-ET cycles was $27.7 \%$. Outcome assessment of frozen-thawed ET cycles in each group (Table 2) demonstrated significantly higher implantation and clinical pregnancy rates in the successful fresh cycle group compared to the unsuccessful fresh cycle group $(35.1 \%$ vs. $13.4 \%$ and $48.9 \%$ vs. $20.8 \%$, respectively; $p<0.001$ for both). Live birth data was available for most of FT-ET pregnancies (164/200). Live birth rate was similarly higher in the successful fresh group $(42.7 \%$ vs. $18.3 \%$, respectively; $p<0.05)$.

Comparison of the FT-ET cycles success rate per patient showed similar significant results in favor of the successful fresh cycle group $(51.3 \%$ vs. $20.8 \%, p<0.001)$. The percentage of patients that achieved at least one pregnancy in their frozen-thawed cycles was significantly higher in the successful fresh cycle group compared with the unsuccessful fresh cycle group $(55.0 \%$ vs. $22.9 \%, p<0.001)$ 
When comparing the first frozen-thawed cycles only, similar significant differences were found with regard to clinical pregnancy rates and live birth rates in favor of the successful fresh cycle group (Table 2).

In a univariate analysis performed, the parameters that were found to be significantly associated with clinical pregnancy in the frozen-thawed cycles were clinical pregnancy in the fresh cycle from the same OPU, age at fresh cycle and number of oocytes retrieved. In order to determine whether previous successful fresh cycle is an independent prognostic factor for successful FT-ET - a multivariate analysis was performed. Logistic regression (Table 3) showed that clinical pregnancy in the fresh cycle, among other factors, remained a significant predictor of success in the consecutive frozen cycle with an adjusted OR of $5.02(95 \% \mathrm{Cl}: 3.12-8.07 ; \mathrm{p}<0.001)$.

Table 3

Multi-variate analysis of factors associated with clinical pregnancy in frozen thawed embryo transfer cycles

\begin{tabular}{|llll|}
\hline Parameter & Adjusted OR & 95\% Cl for OR & P value \\
\hline Clinical pregnancy in fresh cycle & 5.019 & $3.121-8.070$ & $<0.001$ \\
\hline Age at oocyte aspiration (years) & 1.031 & $0.990-1.072$ & 0.148 \\
\hline Age at oocyte aspiration ${ }^{\mathrm{a}}$ & & & \\
\hline Under 35 years & 1.667 & $0.899-3.093$ & 0.105 \\
\hline 35-40 years & 1.867 & $0.931-3.744$ & 0.079 \\
\hline N of oocytes retrieved & 1.041 & $1.008-1.075$ & 0.015 \\
\hline Note: ICSI, Intracytoplasmic sperm injection & & \\
\hline aCompared to patients $>$ 40 years & & & \\
\hline
\end{tabular}

\section{Discussion}

This study aimed to address the possible prediction of FT-ET results with regard to its previous fresh ET cycle from the same OPU. Our results showed consistent, significant differences between patients with a successful fresh ET cycle compared to those with an unsuccessful fresh ET with regard to the success of consecutive FT-ET cycles from the same OPU. This was also demonstrated in the first FT-ET cycle separately. Logistic regression showed this factor to be a significant independent factor, with an adjusted OR of 5.02 .

This is the first study to evaluate this link in embryos cryopreserved using the vitrification method which is the prevalent method applied today in most IVF laboratories. 
As previously reported by van Loendersloot et al. [3] and Lintsen et al. [4], our analyses demonstrated that the number of oocytes retrieved and use of ICSI are independent prognostic factors for successful ET. In addition to the previous studies mentioned, we showed a similar association between previous successful fresh cycle and successful ET. This further establishes these parameters as important prognostic factors that should be evaluated in predicting success of FT-ET.

Our finding of higher pregnancy rates for FT-ET cycles after successful fresh ET are in agreement with that reported by Molloy et al. [11] on 784 patients who had favorable pregnancy outcomes of FT-ET cycles depending on the outcome of the original fresh cycle.

El Toukhy et al. [12] similarly reported double implantation and pregnancy rates in FT-ET cycles using embryos originated from successful fresh ET cycles. Their data was based on 459 fresh cycles. However, both abovementioned studies were carried out prior to the introduction of the vitrification technique. Therefore, they did not assess this phenomenon in embryos frozen by vitrification, rendering them less relevant for IVF programs currently using this technique.

In order to address this issue, our study included only embryos frozen using vitrification. Frozen cycle success rate after vitrification reached $50 \%$ in patients with a previous successful fresh cycle compared with nearly $20 \%$ in patients with a failed fresh cycle.

A systematic review by Rienzy et al. [15] showed that the vitrification technique results in improved survival rates of cryopreserved oocytes and improved clinical pregnancy rates per cycle [16, 17]. Ultrarapid cooling by vitrification enables solidification of cells and extracellular environment into a glass like state without formation of ice eliminating the mechanical injury which may occur during freezing of water particles. We hypothesize that the advantage of this technique over slow freezing may contribute to higher quality frozen-thawed embryos that preserve a "fresh embryo" potential with clinical outcomes as good as fresh embryos. Therefore, when the fresh cycle is successful, the consecutive vitrified frozen thawed embryos from same OPU are of a much higher potential for successful implantation and clinical pregnancy and are only minimally affected by vitrification. This hypothesis is supported by the major difference $(\sim 30 \%)$ in clinical pregnancy rate between our study groups, compared to smaller advantage in clinical pregnancy rates shown in similar studies that investigated this association in embryos frozen by slow freezing.

Besides its retrospective nature, this study has several limitations. During the study period only 122 blastocysts were transferred, Therefore, we were not able to separately analyze the groups according to cleavage stage and blastocysts transfers. Another limitation was the age difference of nearly a year in favor of the successful fresh cycle group. Though this may affect the subsequent frozen cycle's results, our logistic regression demonstrated that successful fresh cycle is an independent factor even when considering age at oocyte retrieval. Clinical pregnancy rates rather than live birth rates were used as the primary outcome due to lack of birth data for some $(\sim 20 \%)$ of the women included in the study. However, in most cases birth data was available and live birth rate was similarly higher in FT-ET following a successful fresh cycle. 
In conclusion, this study shows that in addition to known factors such use of ICSI and number of retrieved oocytes, a successful fresh cycle is a positive independent prognostic factor for a successful FTET cycle of embryos derived from the same OPU. This data may assist physicians facing the challenge of counselling women planning a frozen thawed embryo transfer cycle.

\section{Declarations}

\section{Ethics approval and consent to participate}

The study was approved by the Human Research Ethics Committees of the Hadassah University hospital (IRB 0483-17-HMO) and conforms to the provisions of the declaration of Helsinki.

\section{Consent for publication}

Not applicable.

\section{Availability of data and materials}

The datasets used and/or analyzed during the current study are available from the corresponding author on reasonable request.

\section{Competing interests}

The authors declare that they have no competing interests

\section{Funding}

No funding was received for this study.

\section{Authors contribution}

GK and SH have contributed substantially to the conception and design of the study, analysis and interpretation of data and drafting and revising of the article. NSS has contributed substantially to the acquisition, analysis and interpretation of data and drafting and revision of the article. HHC has contributed substantially to the acquisition, analysis and interpretation of data and drafting and revision of the article. ABM has contributed substantially to the acquisition, analysis and interpretation of data and drafting and revision of the article. AS has contributed substantially to the conception and design of the study, acquisition, analysis and interpretation of data and drafting and revision of the article. All authors read and approved the final manuscript.

Acknowledgments: None

\section{References}


1. Kissen DM, Kulkarni AD, Mneimneh A, Warner L, Boulet SL, Crawford S, etNational ART Surveillance System (NASS) group. Embryo transfer practices and multiple births resulting from assisted reproductive technology: an opportunity for prevention. Fertil Steril. 2015;103:954-61.

2. Penzias A, Bendikson K, Butts S, Coutifaris C, Fossum G, Falcone T, et al. Guidance on the limits to the number of embryos to transfer: a committee opinion. Fertil Steril.2017;107:901-3.

3. van Loendersloot LL, van Wely M, Limpens J, Bossuyt PM, Repping S, van der Veen F. Predictive factors in in vitro fertilization (IVF): a systematic review and meta-analysis. Hum Reprod Update. 2010;16:577-89.

4. Lintsen AM, Eijkemans MJ, Hunault CC, Bouwmans CA, Hakkaart L, Habbema JD, et al.

Predicting ongoing pregnancy chances after IVF and ICSI: a national prospective study. Hum Reprod. 2007;22:2455-62.

5. Hughes EG, King C, Wood EC. A prospective study of prognostic factors in in vitro fertilization and embryo transfer. Fertil Steril. 1989;51:838-44.

6. Erenus M, Zouves C, Rajamahendran P, Leung S, Fluker M, Gomel V. The effect of embryo quality on subsequent pregnancy rates after in vitro fertilization. Fertil Steril 1991;56:707-10.

7. Chuang CC, Chen CD, Chao KH, Chen SU, Ho HN, Yang YS. Age is a better predictor of pregnancy potential than basal follicle-stimulating hormone levels in women undergoing in vitro fertilization. Fertil Steril. 2003;79:63-8.

8. Burke LM, Davenport AT, Russell GB, Deaton JL. Predictors of success after embryo transfer: experience from a single provider. Am J Obstet Gynecol. 2000;182:1001-4.

9. Benchaib M, Braun V, Lornage J, Hadj S, Salle B, Lejeune H, et al. Sperm DNA fragmentation decreases the pregnancy rate in an assisted reproductive technique. Hum Reprod. 2003;18:1023-8.

10. Lin YP, Cassidenti DL, Chacon RR, Soubra SS, Rosen GF, Yee B.

Successful implantation of frozen sibling embryos is influenced by the outcome of the cycle from which they were derived. Fertil Steril. 1995;63:262-7.

11. Molloy D, Doody ML, Breen T. Second time around: a study of patients seeking second assisted reproduction pregnancies. Fertil Steril. 1995;64:546-51.

12. El-Toukhy T, Khalaf Y, Al-Darazi K, O'Mahony F, Wharf E, Taylor A, Braude P. Cryo-thawed embryos obtained from conception cycles have double the implantation and pregnancy potential of those from unsuccessful cycles. Hum Reprod. 2003;18:1313-8.

13. Racowsky C, Vernon M, Mayer J, Ball GD, Behr B, Pomeroy KO, et al. Standardization of grading embryo morphology. J Assist Reprod Genet. 2010;27:437-9. 
14. Simon A, Hurwitz A, Pharhat M, Revel A, Zentner B, Laufer N: A flexible protocol for controlled preparation of the endometrium without prior gonadotropin releasing hormone agonist suppression in women with functioning ovaries undergoing frozen-thawed embryo transfer cycles. Fertil Steril. 1999;71:609-13.

15. Rienzi L, Gracia C, Maggiulli R, LaBarbera AR, Kaser DJ, Ubaldi FM, et al. Oocyte, embryo and blastocyst cryopreservation in ART: systematic review and meta-analysis comparing slow-freezing versus vitrification to produce evidence for the development of global guidance. Hum Reprod Update. 2017;23:139-55.

16. Rama Raju GA, Haranath GB, Krishna KM Prakash GJ, Madan K. Vitrification of human 8-cell embryos, a modified protocol for better pregnancy rates. Reprod Biomed Online. 2005;11:434-7.

17. Debrock S, Peeraer K, Fernandez Gallardo E, De Neubourg D, Spiessens C, D'Hooghe TM. Vitrification of cleavage stage day 3 embryos results in higher live birth rates than conventional slow-freezing: a RCT. Hum Reprod. 2015;30:1820-30. 